\title{
Generalized Polarized Manifolds
}

\author{
Azzouz Awane \\ Equipe de Géométrie Différentielle et Applications \\ Faculté des Sciences Ben M'sik \\ B.P. 7955. Boulevard Driss Harti \\ Casablanca - Maroc. \\ a. awane@yahoo.fr
}

Received: February 8, 2007

Accepted: October 8, 2007

\begin{abstract}
We introduce and develop the notion of generalized Poisson manifolds and analyze their main properties. Several generalized Hamiltonian maps for polarized Poisson manifolds and vectorial Hamiltonian maps for systems in dimension smaller or equal than 4 are given.

Key words: Hamiltonian systems, Poisson manifolds, symplectic structures, generalized Hamiltonian dynamics of Nambu.

2000 Mathematics Subject Classification: 53D10, 53D17, 70G45, $70 \mathrm{H} 05$.
\end{abstract}

\section{Introduction}

$k$-symplectic structures were introduced by the author in $1984([2,21])$, and later generalized by Puta Mircea in 1988 [21]. Analogous properties in this context were analyzed by different authors, see, e.g., [15,20].

A polarized structure on an even dimensional smooth manifold $M$ is a pair $(\theta, E)$ constituted by a closed differential 2-form $\theta$ of maximum rank and by an $n$-codimensional integrable subbundle $E$ of $T M$ which is Lagrangian with respect to the 2 -form $\theta$. Locally, there exists a coordinate system $\left(x^{i}, y^{i}\right)_{1 \leq i \leq n}$ (the Darboux coordinate system) such that

$$
\theta=\sum_{i=1}^{n} d x^{i} \wedge d y^{i},
$$

and the subbundle $E$ is defined by $d y^{1}=\cdots=d y^{n}=0$. 
The notion of a polarized manifold plays an important role in the theory of Kostant-Souriau's geometric quantization, see for e.g.[18,22]. Some important properties are given by A. Weinstein, P. Dazord, J. M. Morvan, P. Molino, P. Libermann.

Let us recall that one of the main motivations which led to introduce the notion of $k$-symplectic structure as an extension of the geometry of polarization ([10]) was to propose a geometric support for the equations of Nambu ([19]), in analogy with the well known symplectic geometry and classical Hamiltonian formalism. Some properties of the Poisson structure subordinate to a $k$-symplectic manifold have led us to introduce the notion of vectorial polarized Poisson structure. For a fixed finite dimensional real vector space $V$, this structure is defined on a foliated manifold $(M, \mathfrak{F})$ by a pair $(\mathfrak{H}(M, \mathfrak{F}), P)$, where $\mathfrak{H}(M, \mathfrak{F})$ is a submodule of the space $\mathcal{C}^{\infty}(M, V)$ of $V$-valued smooth functions on $M$, over the ring of basic functions for the foliation $\mathfrak{F}$, and $P$ is a $\mathcal{C}^{\infty}(M)$-antisymmetric bilinear mapping

$$
P: \bigwedge_{1}(M, V) \times \bigwedge_{1}(M, V) \longrightarrow \mathcal{C}^{\infty}(M, V)
$$

that coincides with the classical case for $V=\mathbb{R}$.

A remarkable feature of the Hamiltonian description of classical dynamics is Liouville's theorem, which states that the volume of phase space occupied by an ensemble of systems is remains invariant. The theorem plays, amongst other things, a fundamental role in statistical mechanics. On the other hand, Hamiltonian dynamics is not the only formalism that makes statistical mechanics possible. Any set of equations which leads to Liouville theorem in a suitably defined phase space will do (provided, of course, that ergodicity may be assumed). Nambu proposes a possible generalization of the Hamiltonian dynamics for a 3-dimensional space.

In this context, $k$-symplectic geometry represents a geometrical tool which comprises differential 2-forms $\theta^{1}, \ldots, \theta^{k}$, such that the Hamiltonian map is $\mathbb{R}^{k}$-valued $H$, the components of which are related to Hamiltonian systems $X_{H}$ by means of the identity

$$
i\left(X_{H}\right) \theta^{p}=-d H^{p},
$$

in order to find Nambu-Hamilton equations preserving the specific features of the classical symplectic geometry.

From this perspective, a $k$-symplectic structure is a $(k+1)$-tuple $\left(\theta^{1}, \ldots, \theta^{k} ; E\right)$ such that $\theta^{1}, \ldots, \theta^{k}$ constitute a non degenerate system vanishing on the tangent vector fields to leaves.

The generalized Darboux theorem shows that there exists about each point $x_{0}$ of $M$ a local coordinate system $\left(x_{i}^{p}, y^{i}\right)_{1 \leq p \leq k, 1 \leq i \leq n}$ defined on a neighborhood such that

$$
\theta_{\mid U}^{p}=d x_{i}^{p} \wedge d y^{i}
$$

and $E$ is given by $d y^{1}=\cdots=d y^{n}=0$.

The study of an exterior system in high dimension, underlines the existence of an infinite number of systems not algebraically equivalent. $k$-symplectic systems 
are defined directly by conditions of regularity; they can be interpreted as models of exterior systems of maximum rank vanishing on the tangent vector fields of the generalized Lagrangian foliation.

In this work, we introduce the notion of a vectorial polarized structure on a manifold $M$. This structure is given by $(\theta, E)$, where $\theta$ is a closed vectorial valued 2 -form on $M$ vanishing on the section of a subbundle $E$. The polarized manifolds and the $k$-symplectic manifolds are particular vectorial polarized manifolds.

\section{Generalized polarized manifolds}

\subsection{Definition}

Let $(M, \mathfrak{F})$ be a foliated manifold, $M$ an $m$-dimensional manifold endowed with a $p$-dimensional foliation, and let $V$ be a real $k$-dimensional vector space.

Let us fix a basis $\left(e_{r}\right)_{1 \leq r \leq k}$ of $V$, and let $\bigwedge_{2}(M, V)=\bigwedge_{2}(M) \otimes V$ be the space of $V$-valued differential two forms; that is, the space of

$$
\theta=\theta^{\alpha} \otimes e_{\alpha}=\theta^{1} \otimes e_{1}+\cdots+\theta^{k} \otimes e_{k}
$$

where $\theta^{1}, \ldots, \theta^{k} \in \bigwedge_{2}(M)$.

We say that $(\theta, E)$ is a vectorial polarized structure, or generalized polarized manifold, on $M$, if the following conditions hold:

(i) The $V$-valued 2 -form $\theta$ is non degenerate, that is,

$$
\forall x \in M, \forall X \in T_{x} M, \quad i(X) \theta=0 \Longrightarrow X=0 .
$$

(ii) Each leaf of $\mathfrak{F}$ is maximal totally isotropic with respect to $\theta$.

\subsection{Hamiltonian systems}

Suppose that $M$ is endowed with a vectorial polarized structure $(\theta ; E)$ and let

$$
j: \mathfrak{X}(M) \longrightarrow \Lambda_{1}(M) \otimes V
$$

be defined by

$$
j(X)=i(X) \theta, \forall X \in \mathfrak{X}(M) .
$$

A vector field $X$ on $M$ is called a vectorial polarized Hamiltonian system if it is an infinitesimal automorphism for the vectorial polarized structure $(\theta ; E)$, that is, if the following conditions are satisfied:

(i) $X$ is foliate;

(ii) $i(X) \theta$ is closed. 
We denote by $\mathfrak{L}(M, \mathfrak{F})$ the $\mathcal{C}^{\infty}(M)$-module of infinitesimal automorphisms of the pair $(\theta, E)$.

Let $X$ be a vectorial polarized Hamiltonian system. By Poincaré lemma, for every $x \in M$, there exists an open neighborhood $U$ of $x$ and a (smooth) mapping $H: U \rightarrow V$ satisfying the relationship

$$
i(X) \theta_{\mid U}=-d H_{\mid U}
$$

Conversely, if a smooth mapping $H: M \rightarrow V$ satisfies

$$
d H=d H^{\alpha} \otimes e_{\alpha} \in j(\mathfrak{L}(M, \mathfrak{F})),
$$

there exists a unique vector field on $M$, denoted $X_{H}$ and called the vectorial polarized Hamiltonian system associated to $H$, such that

$$
i\left(X_{H}\right) \theta=-d H
$$

on $M$. The vector field $X_{H}$ is called a (strongly) Hamiltonian system.

A smooth mapping $H: M \rightarrow V$ satisfying $d H \in j(\mathfrak{L}(M, \mathfrak{F}))$ is called a vectorial polarized Hamiltonian mapping of the vectorial polarized structure $(\theta ; E)$.

\subsection{Poisson bracket of a vectorial polarized structure}

Let $H$ and $K$ be two vectorial polarized Hamiltonian mappings and $X_{H}, X_{K}$ the associated polarized Hamiltonian systems. The Lie bracket $\left[X_{H}, Y_{K}\right]$ is a polarized Hamiltonian system. More precisely, the mapping of $M$ into $V$ defined by

$$
\{H, K\}=\theta\left(X_{H}, X_{K}\right)=\theta^{\alpha}\left(X_{H}, X_{K}\right) e_{\alpha}
$$

satisfies

$$
\left[X_{H}, X_{K}\right]=X_{\{H, K\}} .
$$

The mapping $\{H, K\}$ is called the (vectorial) Poisson bracket of the (vectorial) Hamiltonian mappings $H$ and $K$. We denote the space of all vectorial polarized Hamiltonian mappings by $\mathfrak{H}(M, \mathfrak{F}, V)$.

\section{Polarized manifolds}

A real polarization on $M$ is a vectorial polarization $(\theta, E)$ such that $m=2 p$ and $V=\mathbb{R}$.

Theorem 2.1 (Darboux theorem). Every point of $M$ has an open neighborhood $U$ with local coordinates system $\left(x^{1}, \ldots, x^{n}, y^{1}, \ldots, y^{n}\right)$ such that

$$
\theta=d x^{1} \wedge d y^{1}+\cdots+d x^{n} \wedge d y^{n}
$$

and $\mathfrak{F}$ is defined by equations $d y^{1}=\cdots=d y^{n}=0$. 
And with respect to an adapted coordinates system $\left(x^{1}, \ldots, x^{n}, y^{1}, \ldots, y^{n}\right)$, the polarized Hamiltonian mapping $H$ takes the form

$$
H=\sum_{i=1}^{n} a_{i}\left(y^{1}, \ldots, y^{n}\right) x^{i}+b\left(y^{1}, \ldots, y^{n}\right)
$$

where $a_{1}, \ldots, a_{n}, b$ are basic functions for $\mathfrak{F}$. Recall that, by the symplectic duality $\zeta: X \mapsto i(X) \theta$ between the tangent bundle $T M$ and the cotangent bundle $T^{*} M$, we associate to $\theta$ a non degenerate bivector $P$ (the Poisson tensor) defined by

$$
P(\alpha, \beta)=\theta\left(\zeta^{-1}(\alpha), \zeta^{-1}(\beta)\right) \quad \text { for all } \alpha, \beta \in \bigwedge_{1}(M),
$$

and we have an antisymmetric linear mapping $\underline{P}: \bigwedge_{1}(M) \rightarrow \mathfrak{X}(M)$, given by

$$
\langle\beta, \underline{P}(\alpha)\rangle=P(\alpha, \beta) .
$$

\section{3. $k$-symplectic manifolds}

A $k$-symplectic structure on $M$ is a vectorial polarization $(\theta, E)$ such that $m=n(k+1)$ and $p=n k$.

\subsection{Canonical $k$-symplectic structure on $\mathbb{R}^{n(k+1)}$}

Consider $\mathbb{R}^{n(k+1)}$ endowed with its Cartesian coordinates $\left(x_{i}^{\alpha}, y^{i}\right)_{1 \leq \alpha \leq k, 1 \leq i \leq n}$. Let $E$ be the subbundle of $T \mathbb{R}^{n(k+1)}$ defined by the equations

$$
d y^{1}=0, \ldots, d y^{n}=0
$$

and

$$
\theta=\theta^{\alpha} \otimes e_{\alpha}=\left(d x_{i}^{\alpha} \wedge d y^{i}\right) \otimes e_{\alpha}
$$

The pair $(\theta, E)$ defines a $k$-symplectic structure on $\mathbb{R}^{n(k+1)}$ called the canonical $k$-symplectic structure. This structure induces a natural $k$-symplectic structure on the torus $\mathbb{T}^{n(k+1)}$.

\subsection{The generalized Darboux theorem}

Let $M$ be an $n(k+1)$-dimensional manifold. If $\left(\theta=\theta^{\alpha} \otimes e_{\alpha}, E\right)$ is a $k$-symplectic structure on $M$ then for every point $p$ of $M$ there exists an open neighborhood $U$ of $M$ containing $p$ endowed with local coordinates $\left(x_{i}^{\alpha}, y^{i}\right)_{1 \leq \alpha \leq k, 1 \leq i \leq n}$ called an adapted coordinate system, such that the $V$-valued differential form $\theta$ is represented on $U$ by

$$
\theta=\theta^{\alpha} \otimes e_{\alpha}=\left(d x_{i}^{\alpha} \wedge d y^{i}\right) \otimes e_{\alpha},
$$

and $E$ is defined by the equations $d y^{1}=0, \ldots, d y^{n}=0$. 
Proposition 3.1. Let $H=\left(H^{\alpha}\right)_{1 \leq \alpha \leq k}$ be a vectorial polarized Hamiltonian mapping and let $X_{H}$ be the associated polarized Hamiltonian system. With respect to an adapted coordinates system $\left(x_{i}^{\alpha}, y^{i}\right)_{1 \leq \alpha \leq k, 1 \leq i \leq n}$, the components $H^{p}$ of $H$ and $X_{H}$ can be written as

$$
H^{\alpha}=x_{j}^{\alpha} f^{j}\left(y^{1}, \ldots, y^{n}\right)+g^{\alpha}\left(y^{1}, \ldots, y^{n}\right)
$$

and

$$
\begin{aligned}
X_{H}= & -\left(x_{j}^{\alpha} \frac{\partial f^{j}}{\partial y^{s}}\left(y^{1}, \ldots, y^{n}\right)+\frac{\partial g^{\alpha}}{\partial y^{s}}\left(y^{1}, \ldots, y^{n}\right)\right) \frac{\partial}{\partial x_{s}^{\alpha}} \\
& +f^{s}\left(y^{1}, \ldots, y^{n}\right) \frac{\partial}{\partial y^{s}}
\end{aligned}
$$

where $f^{j}$ and $g^{\alpha}$ are smooth basic functions on $U$.

Remark 3.2. Assume that $k \geq 2$. It follows from the proof of the previous proposition that, if the Pfaffian forms $i(X) \theta^{1}, \ldots, i(X) \theta^{k}$ are closed (or equivalently $L_{X} \theta^{1}=\cdots=$ $\left.L_{X} \theta^{k}=0\right)$, then the vector field $X$ is necessarily an infinitesimal automorphism of $\mathfrak{F}$.

With respect to an adapted coordinate system $\left(x_{i}^{\alpha}, y^{i}\right)_{1 \leq \alpha \leq k, 1 \leq i \leq n}$, the components $\{H, K\}^{\alpha}$ of $\{H, K\}$ are given by

$$
\{H, K\}^{\alpha}=\sum_{s=1}^{n}\left(\frac{\partial H^{\alpha}}{\partial x_{s}^{\alpha}} \frac{\partial K^{\alpha}}{\partial y^{s}}-\frac{\partial H^{\alpha}}{\partial y^{s}} \frac{\partial K^{\alpha}}{\partial x_{s}^{\alpha}}\right),
$$

Let $\mathfrak{H}(M)$ be the set of Hamiltonian mappings of the $k$-symplectic structure $\left(\theta^{1}, \ldots, \theta^{k} ; E\right)$. The correspondence $(H, K) \rightarrow\{H, K\}$, of $\mathfrak{H}(M) \times \mathfrak{H}(M)$ into $\mathfrak{H}(M)$, is a skew-symmetric $\mathbb{R}$-bilinear mapping satisfying the Jacobi identity.

Proposition 3.3. $(\mathfrak{H}(M),\{\}$,$) is an infinite-dimensional Lie algebra.$

\section{Nambu's statistical mechanics}

Let $(x, y, z)$ be a triplet of dynamical variables (a canonical triplet) which spans a 3 -dimensional phase space $M$. This is a formal generalization of conventional phase space spanned by a canonical pair $(p, q)$. Next, we will introduce two functions $H$ and $G$ depending on $(x, y, z)$ which serve as a pair of "Hamiltonians" to determine the motion of points in phase space. More precisely Nambu has postulated the following Hamilton equations:

$$
\left\{\begin{array}{l}
\frac{d x}{d t}=\frac{D(H, G)}{D(y, z)} \\
\frac{d y}{d t}=\frac{D(H, G)}{D(z, x)} \\
\frac{d z}{d t}=\frac{D(H, G)}{D(x, y)}
\end{array}\right.
$$


where $D(H, G) / D(y, z)$ denote the Jacobian

$$
\frac{D(H, G)}{D(y, z)}=\frac{\partial H}{\partial y} \frac{\partial G}{\partial z}-\frac{\partial H}{\partial z} \frac{\partial G}{\partial y} .
$$

The above equations are called Nambu's equations of motion, and the vector field whose integral curves are given by Nambu's equations of motion will be denoted by $X_{(H, G)}^{n}$ and called the dynamical system of Nambu.

Consider the space $M=\mathbb{R}^{3}$ endowed with its canonical 2-symplectic structure $\left(\theta^{1}, \theta^{2} ; E\right)$ defined by

$$
\left\{\begin{array}{l}
\theta^{1}=d x \wedge d z, \\
\theta^{2}=d y \wedge d z, \\
E=\text { ker } d z .
\end{array}\right.
$$

The Hamiltonian mapping of the 2-symplectic structure is the mapping

$$
H: M \longrightarrow \mathbb{R}^{2}
$$

whose components are given by

$$
\left\{\begin{array}{l}
H^{1}=f(z) x+g^{1}(z), \\
H^{2}=f(z) y+g^{2}(z),
\end{array}\right.
$$

where $f, g^{1}$, and $g^{2}$ are smooth real functions depending only on the variable $z$. The integral curves of the Hamiltonian system $X_{H}$ of the 2-symplectic structure are given by the following equations:

and

$$
\begin{aligned}
& \frac{d x}{d t}=-\frac{\partial H^{1}}{\partial z}, \\
& \frac{d y}{d t}=-\frac{\partial H^{2}}{\partial z},
\end{aligned}
$$

$$
\frac{d z}{d t}=\frac{\partial H^{1}}{\partial x}=\frac{\partial H^{2}}{\partial y} .
$$

Theorem 4.1. Let $H=\left(H^{1}, H^{2}\right)$ be a Hamiltonian mapping of the 2-symplectic structure, where $H^{1}=f(z) x+g^{1}(z)$ and $\left.H^{2}=f(z) y+g^{2}(z)\right)$. Then the Hamiltonian system $X_{H}$ and the dynamical system of Nambu $X_{H}^{n}$ are related by

$$
X_{H}^{n}=f(z) X_{H} .
$$

Corollary 4.2. The mapping

$$
(f(z))^{-1} H=\left(x+h^{1}(z), y+h^{2}(z)\right)
$$

is a solution of Nambu's equations of motions on a domain where $f(z)$ is a nonvanishing function and

$$
h^{1}(z)=(f(z))^{-1} g^{1}(z), \quad h^{2}(z)=(f(z))^{-1} g^{2}(z) .
$$




\section{Vectorial polarized Poisson manifolds}

\subsection{Definition}

Let $(M, \mathfrak{F})$ be a foliated manifold, let $M$ be an $n$-dimensional manifold endowed with a $p$-dimensional foliation, and let $V$ be a $k$-dimensional real vector space.

Let us fix a basis $\left(e_{r}\right)_{1 \leq r \leq k}$ of $V$ with dual basis $\left(\omega^{r}\right)_{1 \leq r \leq k}$, and let $\bigwedge_{1}(M, V)=$ $\bigwedge_{1}(M) \otimes V$ be the space of $\bar{V}$-valued differential forms of degree 1 , that is, the space of

$$
\alpha=\alpha^{1} \otimes e_{1}+\cdots+\alpha^{k} \otimes e_{k}
$$

where $\alpha^{1}, \ldots, \alpha^{k} \in \bigwedge_{1}(M)$. Locally, on an open neighborhood $U$ endowed with local coordinates system $\left(x^{1}, \ldots, x^{n}\right)$, each element $\alpha \in \bigwedge_{1}(M, V)$ has the form

$$
\alpha_{\mid U}=\sum_{r=1}^{k} \sum_{i=1}^{n} \alpha_{i}^{r} d x^{i} \otimes e_{r}
$$

where $\alpha_{i}^{r}: U \rightarrow \mathbb{R}$ are smooth mappings.

We denote by $E_{V}^{o}$ the annihilator of the subbundle $E$ in $\bigwedge_{1}(M, V)$, that is, the space of $V$-valued 1 -forms on $M$ vanishing on the cross sections of $E$.

Definition 5.1. Let $(M, \mathfrak{F})$ be a foliated manifold, let $\mathfrak{H}(M, \mathfrak{F})$ be a submodule of $\mathcal{C}^{\infty}(M, V)$ over the ring $\mathfrak{B}(M, \mathfrak{F})$ of basic functions for the foliation $\mathfrak{F}$ and let

$$
P: \bigwedge_{1}(M, V) \times \bigwedge_{1}(M, V) \longrightarrow \mathcal{C}^{\infty}(M, V)
$$

be an antisymmetric $\mathcal{C}^{\infty}(M)$-bilinear mapping. We say that $(\mathfrak{H}(M, \mathfrak{F}), P)$ is a vectorial polarized Poisson structure on $M$, if the following properties hold:

(i) $P(\alpha, \beta)=0$ for all $\alpha, \beta \in E_{V}^{o}$,

(ii) for all $H, K \in \mathfrak{H}(M, \mathfrak{F}), P(d H, d K) \in \mathfrak{H}(M, \mathfrak{F})$,

(iii) the correspondence $(H, K) \rightarrow\{H, K\}=P(d H, d K)$, from $\mathfrak{H}(M, \mathfrak{F}) \times \mathfrak{H}(M, \mathfrak{F})$ with values in $\mathfrak{H}(M, \mathfrak{F})$, gives to $\mathfrak{H}(M, \mathfrak{F})$ a Lie algebra law,

(iv) each $H \in \mathfrak{H}(M, \mathfrak{F})$ corresponds to a vector field $X_{H}$ such that

$$
\left\langle d K, X_{H}\right\rangle=\{H, K\},
$$

for all $K \in \mathfrak{H}(M, \mathfrak{F})$.

$P$ will be called a vectorial polarized Poisson tensor.

Let us consider an open neighborhood $U$ of $M$ endowed with an adapted local coordinates system $\left(x^{1}, \ldots, x^{p}, y^{1}, \ldots, y^{q}\right)$. Since $P$ is zero on the annihilator $E_{V}^{o}$ of the subbundle $E$ in $\bigwedge_{1}(M, V)$, then the tensor $P$ has the form

$$
P=A_{p q}^{i j r}\left(\left(\frac{\partial}{\partial x^{i}} \otimes \omega^{p}\right) \wedge\left(\frac{\partial}{\partial x^{j}} \otimes \omega^{q}\right)\right) \otimes e_{r}+B_{p q}^{i j r}\left(\left(\frac{\partial}{\partial x^{i}} \otimes \omega^{p}\right) \wedge\left(\frac{\partial}{\partial y^{j}} \otimes \omega^{q}\right)\right) \otimes e_{r}
$$


where $A_{p q}^{i j r}, B_{p q}^{i j r}: U \rightarrow \mathbb{R}$ are differential mappings. The Jacobi identity implies that

$$
\begin{aligned}
& \frac{\partial A_{u v}^{a b r}}{\partial x^{l}} A_{r w}^{l c v}-\frac{\partial A_{u v}^{a b r}}{\partial y^{m}} B_{w r}^{c m v}+\frac{\partial A_{v w}^{b c r}}{\partial x^{l}} A_{r u}^{l a v} \\
&-\frac{\partial A_{v w}^{b c r}}{\partial y^{m}} B_{u r}^{a m v}+\frac{\partial A_{w u}^{c a r}}{\partial x^{l}} A_{r v}^{l b v}-\frac{\partial A_{w u}^{c a r}}{\partial y^{m}} B_{v r}^{b m v}=0, \\
& \frac{\partial A_{u v}^{a b r}}{\partial x^{l}} B_{r w}^{l c v}+\frac{\partial B_{v w}^{b c r}}{\partial x^{l}} A_{r u}^{l a v}-\frac{\partial B_{v w}^{b c r}}{\partial y^{m}} B_{u r}^{a m v}-\frac{\partial B_{u w}^{a c r}}{\partial x^{l}} A_{r v}^{l b v}+\frac{\partial B_{u w}^{a c r}}{\partial y^{m}} B_{v r}^{b m v}=0, \\
& \frac{\partial B_{u v}^{a b v}}{\partial x^{l}} A_{r v}^{l b v}-\frac{\partial B_{u w}^{a c r}}{\partial x^{l}} B_{r v}^{l b v}=0 .
\end{aligned}
$$

For each element $\alpha \in \bigwedge_{1}(M, V)$ we can associate a $\mathcal{C}^{\infty}(M)$-linear mapping

$$
P(\alpha, \cdot): \bigwedge_{1}(M, V) \longrightarrow \mathcal{C}^{\infty}(M, V)
$$

such that $P(\alpha, \cdot)(\beta)=P(\alpha, \beta)$ for each $\beta \in \bigwedge_{1}(M, V)$. The linear mapping $P(\alpha, \cdot)$ coincides with the vector field $\underline{P}(\alpha)$ for $k=1$.

\section{Model vectorial polarized Poisson manifolds}

Let us consider the model space $\mathbb{R}^{n}=\mathbb{R}^{p} \times \mathbb{R}^{q}$ endowed with the $p$-dimensional model foliation $\mathfrak{F}$ defined by the equations $d y^{1}=\cdots=d y^{q}=0$, where $\left(x^{i}, y^{j}\right)$, with $i=1, \ldots, p$ and $j=1, \ldots, q$, are the Cartesian coordinates system and let $V=\mathbb{R}^{k}$ be the real space where the canonical basis $\left(e_{r}\right)_{1 \leq r \leq k}$ with dual basis $\left(\omega^{r}\right)_{1 \leq r \leq k}$ is fixed.

Let $(\mathfrak{H}(M, \mathfrak{F}), P)$ be a vectorial polarized Poisson structure on $\mathbb{R}^{n}$. The vectorial polarized Poisson bivector $P$ takes the form (1) and satisfies the Jacobi identity (2), and $\mathfrak{H}(M, \mathfrak{F})$ is a submodule of $\mathcal{C}^{\infty}\left(\mathbb{R}^{n}, V\right)$ over the ring $\mathfrak{B}\left(\mathbb{R}^{n}, \mathfrak{F}\right)$ of basic functions. Now we give some examples of polarized Poisson structures widening the space of vectorial polarized Hamiltonian mappings subordinate to $k$-symplectic manifolds.

(i) Let $\mathfrak{H}\left(\mathbb{R}^{n}, \mathfrak{F}\right)$ be the $\mathfrak{B}(M, \mathfrak{F})$-submodule of $\mathcal{C}^{\infty}\left(\mathbb{R}^{n}, V\right)$ spanned by $e_{1}, \ldots, e_{k}$. Thus $\mathfrak{H}\left(\mathbb{R}^{n}, \mathfrak{F}\right)=\mathfrak{B}\left(\mathbb{R}^{n}, \mathfrak{F}\right) \times \cdots \times \mathfrak{B}\left(\mathbb{R}^{n}, \mathfrak{F}\right)$ ( $k$ times) and for all vectorial polarized Poisson bivector $P$, the associated Lie algebra $(\mathfrak{B}(M, \mathfrak{F}),\{\}$,$) is$ Abelian.

(ii) For $V=\mathbb{R}^{2}$ we consider the $\mathfrak{B}(M, \mathfrak{F})$-submodule $\mathfrak{H}\left(\mathbb{R}^{n}, \mathfrak{F}\right)$ of $\mathcal{C}^{\infty}\left(\mathbb{R}^{n}, V\right)$ spanned by the mappings

$$
\begin{array}{rrr}
X^{I}:(x, y) & \longmapsto x^{I} e_{1} & (I=1, \ldots, p), \\
X^{i j}:(x, y) \longmapsto x^{i} x^{j} e_{2} & (i, j=1, \ldots, p),
\end{array}
$$


and by the vectors $e_{1}, e_{2}$. The components $H^{1}$ and $H^{2}$ of each element $H$ of $\mathfrak{B}(M, \mathfrak{F})$ take the form

$$
\begin{aligned}
& H^{1}=\sum_{i=1}^{p} f_{i}\left(y^{1}, \ldots, y^{q}\right) x^{i}+g^{1}\left(y^{1}, \ldots, y^{q}\right) \quad(i=1, \ldots, p), \\
& H^{2}=\sum_{i, j=1}^{p} f_{i j}\left(y^{1}, \ldots, y^{q}\right) x^{i} x^{j}+g^{2}\left(y^{1}, \ldots, y^{q}\right) \quad(i, j=1, \ldots, p),
\end{aligned}
$$

where $f_{i}, f_{i j}, g^{1}, g^{2} \in \mathfrak{B}(M, \mathfrak{F})$.

(iii) Suppose that $p=m k$. We denote by $(x, y)=\left(x^{r a}, y^{1}, \ldots, y^{q}\right)_{1<a<m, 1<r<k}$ the Cartesian coordinates system of $\mathbb{R}^{n}$. Let $\mathfrak{H}\left(\mathbb{R}^{n}, \mathfrak{F}\right)$ be the $\mathfrak{B}(M, \overline{\mathfrak{F}})$-submodule of $\mathcal{C}^{\infty}\left(\mathbb{R}^{n}, V\right)$ spanned by the mappings

$$
X^{a}:(x, y) \longmapsto \sum_{r=1}^{k} x^{r a} e_{r} \quad(a=1, \ldots, m)
$$

and by the vectors $e_{1}, \ldots, e_{k}$. The component $H^{r}$ of each element $H$ of $\mathfrak{H}(M, \mathfrak{F})$ takes the form

$$
H^{r}=\sum_{a=1}^{m} f_{a}\left(y^{1}, \ldots, y^{n}\right) x^{r a}+g^{r}\left(y^{1}, \ldots, y^{n}\right) \quad(r=1, \ldots, k),
$$

where $f_{a}, g^{r} \in \mathfrak{B}(M, \mathfrak{F})$.

(iv) In the previous notations, we consider the $\mathfrak{B}(M, \mathfrak{F})$-submodule $\mathfrak{H}\left(\mathbb{R}^{n}, \mathfrak{F}\right)$ of $\mathcal{C}^{\infty}\left(\mathbb{R}^{n}, V\right)$ spanned by the mappings

$$
X^{a b}:(x, y) \longmapsto \sum_{r=1}^{k} x^{r a} x^{r b} e_{r} \quad(a, b=1, \ldots, m),
$$

and by the vectors $e_{1}, \ldots, e_{k}$. The component $H^{r}$ of each element $H$ of $\mathfrak{B}(M, \mathfrak{F})$ has the form

$$
H^{r}=\sum_{a, b=1}^{m} f_{a b}\left(y^{1}, \ldots, y^{n}\right) x^{r a} x^{r b}+b^{r}\left(y^{1}, \ldots, y^{n}\right) \quad(r=1, \ldots, k),
$$

where $f_{a b}, g^{r} \in \mathfrak{B}(M, \mathfrak{F})$.

(v) In the previous notations, we consider the $\mathfrak{B}(M, \mathfrak{F})$-submodule $\mathfrak{H}\left(\mathbb{R}^{n}, \mathfrak{F}\right)$ of 
$\mathcal{C}^{\infty}\left(\mathbb{R}^{n}, V\right)$ spanned by the mappings

$$
\begin{aligned}
X^{a}:(x, y) & \longmapsto \sum_{r=1}^{k} x^{r a} e_{r} & (a=1, \ldots, m), \\
X^{a b}:(x, y) & \longmapsto \sum_{r=1}^{k} x^{r a} x^{r b} e_{r} & (a, b=1, \ldots, m)
\end{aligned}
$$

and by the vectors $e_{1}, \ldots, e_{k}$. The components $H^{r}$ of each element $H$ of $\mathfrak{B}(M, \mathfrak{F})$ take the form

$$
H^{r}=\sum_{a=1}^{m} f_{a}\left(y^{1}, \ldots, y^{n}\right) x^{r a}+\sum_{a, b=1}^{m} f_{a b}\left(y^{1}, \ldots, y^{n}\right) x^{r a} x^{r b}+b^{r}\left(y^{1}, \ldots, y^{n}\right),
$$

where $r=1, \ldots, k$ and $f_{a}, f_{a b}, g^{r} \in \mathfrak{B}(M, \mathfrak{F})$.

\section{Local models of vectorial polarized systems}

For polarized manifolds and $k$-symplectic manifolds, there is a unique model: the Darboux model and its generalization.

In the case of the vectorial polarized systems, there is not a unique model.

Here we give, some local models of the vectorial polarized systems and the corresponding vectorial polarized Hamiltonian mappings, in the some cases where the dimension of the space is less or equal than 4.

\subsection{For $k=2$ and $m=3$}

If every $\mathbb{R}^{2}$-valued form $\theta=\theta^{1} \otimes e_{1}+\theta^{2} \otimes e_{2}$ in $\mathbb{R}^{3}$ admits a maximal solutions of dimension 2 , then it is a 2 -symplectic system and can be written under the following local form:

$$
\left\{\begin{array}{l}
\theta^{1}=d x^{1} \wedge d x^{3} \\
\theta^{32}=d x^{2} \wedge d x^{3}
\end{array}\right.
$$

\subsection{For $k=3$ and $m=3$}

Consider the $\mathbb{R}^{3}$-valued form in $\mathbb{R}^{3}$ given by

$$
\theta=\theta^{1} \otimes e_{1}+\theta^{2} \otimes e_{2}+\theta^{3} \otimes e_{3},
$$

with rank 3 , such that the system $\left\{\theta^{1}, \theta^{2}, \theta^{3}\right\}$ is not algebraically equivalent to the 2 -system. Then, locally, this system is algebraically equivalent to the following model:

$$
\left\{\begin{array}{l}
\theta^{1}=d x^{2} \wedge d x^{3}, \\
\theta^{2}=d x^{3} \wedge d x^{1}, \\
\theta^{3}=d x^{1} \wedge d x^{2} .
\end{array}\right.
$$


This system has a 1-dimensional maximal solutions.

The vectorial polarized Hamiltonian mappings take the following expressions:

$$
\left\{\begin{array}{l}
H^{1}=a x^{2} x^{3}+b_{3} x^{2}-b_{2} x^{3}+\alpha_{1}, \\
H^{2}=-a x^{1} x^{3}+b_{1} x^{3}-b_{3} x^{1}+\alpha_{2}, \\
H^{3}=-a x^{1} x^{2}+b_{2} x^{1}-b_{1} x^{2}+\alpha_{3} .
\end{array}\right.
$$

where $a, b_{1}, b_{2}, b_{3}, \alpha_{1}, \alpha_{2}, \alpha_{3}$ are real numbers.

\subsection{For $k=2$ and $m=4$}

Let $\theta=\theta^{1} \otimes e_{1}+\theta^{2} \otimes e_{2}$ be a $\mathbb{R}^{2}$-valued form in $\mathbb{R}^{4}$ with maximum rank and with maximal solutions of dimension 2 . Then locally we have

$$
\left\{\begin{array}{l}
\theta^{1}=d x^{1} \wedge d y^{1}, \\
\theta^{2}=d x^{2} \wedge d y^{2} .
\end{array}\right.
$$

This system has a 2-dimensional maximal solutions (the foliation defined by $d y^{1}=$ $\left.d y^{2}=0\right)$.

The vectorial polarized Hamiltonians mappings take the following expressions:

$$
\left\{\begin{array}{l}
H^{1}=f^{1}\left(y^{1}, y^{2}\right) x^{1}+g^{1}\left(y^{1}, y^{2}\right), \\
H^{2}=f^{2}\left(y^{1}, y^{2}\right) x^{2}+g^{2}\left(y^{1}, y^{2}\right),
\end{array}\right.
$$

where $f^{1}, f^{2}, g^{1}, g^{2}$ are basic functions for the foliation defined by $d y^{1}=d y^{2}=0$.

\subsection{For $k=3$ and $m=4$}

We consider only

(i) The 3-symplectic system

$$
\left(S^{1}\right) \quad\left\{\begin{array}{l}
\theta^{1}=d x^{1} \wedge d y \\
\theta^{2}=d x^{2} \wedge d y \\
\theta^{3}=d x^{3} \wedge d y
\end{array}\right.
$$

(ii) The system

$$
\left(S^{2}\right)\left\{\begin{array}{l}
\theta^{1}=d x^{1} \wedge d y^{2}+d x^{2} \wedge d y^{1}, \\
\theta^{2}=d x^{1} \wedge d y^{1}, \\
\theta^{3}=d x^{2} \wedge d y^{2} .
\end{array}\right.
$$

This system has a 2-dimensional maximal solutions (the foliation defined by $\left.d y^{1}=d y^{2}=0\right)$. 
The vectorial polarized Hamiltonian mappings take the following expressions:

$$
\left\{\begin{array}{l}
H^{1}=f^{1}\left(y^{1}, y^{2}\right) x^{1}+g^{1}\left(y^{1}, y^{2}\right), \\
H^{2}=f^{2}\left(y^{1}, y^{2}\right) x^{2}+g^{2}\left(y^{1}, y^{2}\right), \\
H^{3}=f^{2}\left(y^{1}, y^{2} t\right) x^{1}-f^{1}\left(y^{1}, y^{2}\right) x^{2}+g^{3}\left(y^{1}, y^{2}\right) .
\end{array}\right.
$$

where $f^{1}, f^{2}, f^{3}, g^{1}, g^{2}, g^{3}$ are basic functions for the foliation defined by $d y^{1}=d y^{2}=0$.

(iii) The system

$$
\left(S^{3}\right)\left\{\begin{array}{l}
\theta^{1}=d x^{1} \wedge d x^{2}+d x^{3} \wedge d x^{4}, \\
\theta^{2}=d x^{1} \wedge d x^{3}-d x^{2} \wedge d x^{4}, \\
\theta^{3}=d x^{1} \wedge d x^{4}+d x^{2} \wedge d x^{3} .
\end{array}\right.
$$

Locally, this system has a 1-dimensional maximal solution.

The vectorial polarized Hamiltonian mappings take the following expressions:

$$
\left\{\begin{array}{l}
H^{1}\left(x_{1}, x_{2}, x_{3}, x_{4}\right)=a x_{1}+b x_{2}+c x_{3}+d x_{4}+\alpha_{1}, \\
H^{2}\left(x_{1}, x_{2}, x_{3}, x_{4}\right)=-d x_{1}-c x_{2}+b x_{3}+a x_{4}+\alpha_{2}, \\
H^{3}\left(x_{1}, x_{2}, x_{3}, x_{4}\right)=c x_{1}-d x_{2}-a x_{3}+b x_{4}+\alpha_{3} .
\end{array}\right.
$$

where $a, b, c, d, \alpha_{1}, \alpha_{2}, \alpha_{3}$ are real numbers.

\section{References}

[1] J. M. Ancoechea Bermúdez, Sobre la rigidez de álgebras de Lie, Ph.D. Thesis, Universidad Complutense, Madrid, 1984.

[2] A. Awane, Sur une généralisation des structures symplectiques, Ph.D. Thesis, Université Louis Pasteur, Strasbourg, 1984.

[3] _ Systèmes extérieures k-symplectiques, Rend. Sem. Mat. Univ. Politec. Torino 56 (1988), no. $1,65-80$.

[4] _ k-symplectic structures, J. Math. Phys. 33 (1992), no. 12, 4046-4052.

[5] _ Structures k-symplectiques, Ph.D. Thesis, Université de Haute Alsace, Mulhouse, 1992.

[6] G G-espaces k-symplectiques homogènes, J. Geom. Phys. 13 (1994), no. 2, 139-157 (French, with English summary).

[7] Some affine properties of the k-symplectic manifolds, Beiträge Algebra Geom. 39 (1998), no. 1, 75-83.

[8] A. Awane, Polarized Poisson manifolds and its generalizations, Afr. J. Math. Phys. 2 (2005), no. $1,57-64$.

[9] A. Awane, A. Chkiriba, and M. Goze, k-symplectic affine algebras, Rend. Sem. Fac. Sci. Univ. Cagliari 74 (2004), no. 1-2, 1-16.

[10] A. Awane and M. Goze, Pfaffian systems, k-symplectic systems, Kluwer Academic Publishers, Dordrecht, 2000 . 
[11] E. Cartan, Les systèmes de Pfaff à cinq variables et les équations aux dérivées partielles du second ordre, Ann. Sci. École Norm. Sup. (3) 27 (1910), 109-192.

[12] C. Godbillon, Géométrie différentielle et mécanique analytique, Hermann, Paris, 1969.

[13] _ Feuilletages: Etude géométrique, Progress in Mathematics, vol. 98, Birkhauser, Basel, 1991.

[14] M. de León, I. Méndez, and M. Salgado, Regular p-almost cotangent structures, J. Korean Math. Soc. 25 (1988), no. 2, 273-287.

[15] M. de León, J. Main-Solano, and J. C. Marrero, A geometrical approach to classical field theories: A constraint algorithm for singular theories, available at http://www.eco.ub.es/ $\sim$ jmarin/ CONFIEL.pdf.

[16] P. Libermann and C. M. Marle, Géométrie symplectique: Bases théorique de la Mécanique classique., U.E.R. de Mathématiques 1-4;, L.A. de la C.N.R.S. 212;, E.R.A. de la C.N.R.S. 944, 1020, 1021.

[17] P. Molino, Géométrie globale des feuilletages riemanniens, Nederl. Akad. Wetensch. Indag. Math. 44 (1982), no. 1, 45-76.

[18] _ Géométrie de polarisation, Feuilletages et quantification géométrique, Travaux en cours, Hermann, 1984, pp. 37-53.

[19] Y. Nambu, Generalized Hamiltonian dynamics, Phys. Rev. D (3) 7 (1973), no. 8.

[20] L. K. Norris, The n-symplectic algebra of observables in covariant Lagrangian field theory, J. Math. Phys., to appear.

[21] M. Puta, Some Remarks on the k-symplectic manifolds, Tensor (N.S.) 47 (1988), no. 2, 109-115.

[22] D. J. Simms and N. M. J. Woodhouse, Some Remarks on the $k$-symplectic manifolds: Lecture on geometric quantization, Lecture Notes in Physics, vol. 53, 1976. 